\title{
Altbewährtes und Neues - Update Krampfaderbehandlung 2016
}

\author{
Well-Tried and New Ones - Update Varicose Vein Treatment \\ 2016
}

\author{
1,2Heiko Uthoff, ${ }^{2}$ Luca Spinedi, ${ }^{3}$ Thomas Lattmann, ${ }^{4}$ Pavel Broz, ${ }^{2}$ Daniel Staub
}

Gefässpraxis am See, St. Anna im Bahnhof, Luzern'; Angiologie, Universitätsspital Basel²; Gefässchirurgie, Kantonsspital Winterthur³; Angiologie, Spital Limmattal, Schlieren/Zürich ${ }^{4}$

Zusammenfassung: Venöse Beinbeschwerden sind häufig, werden aber auch häufig unterschätzt. Die Behandlung von Krampfadern hat mit der Entwicklung von effektiven, minimal-invasiven endovenösen Methoden in den letzten Jahren grosse Fortschritte gemacht. Grundlage jeder Behandlung ist weiterhin eine kompetente Anamnese, eine klinische Untersuchung und eine Duplexsonografie. Ambulante endovenöse Verfahren werden bei mindestens gleich hoher Effektivität, aber geringeren Nebenwirkungen die traditionelle Chirurgie als Methode der ersten Wahl in der Behandlung von Stammvenen zunehmend ablösen. Seit 1. Januar 2016 sind die endovenösen thermischen Verfahren (Laser/Radiofrequenz) zur Stammvenentherapie durch das Bundesamt für Gesundheit anerkannt und vergütungspflichtige Leistungen in der Grundversorgung.

Alle Methoden haben Vor-und Nachteile bzw. Limitationen, eine individuelle Auswahl anhand der vorhandenen Symptome, anatomischen Befunde und Patientenpräferenzen sind für ein optimales Ergebnis und maximale Patientenzufriedenheit elementar.

Schlüsselwörter: Krampfadern - Varikose - endovenöse Behandlung - Laser - Radiofrequenz

\begin{abstract}
Venous leg symptoms are frequent, but their relevance is frequently underestimated. With the introduction of effective, minimal-invasive endovenous treatment modalities the treatment of varicose veins has recently made major advances. The basis of every treatment decision is a competent anamnesis, clinical investigation and duplex scan. Outpatient varicose vein treatment using endovenous methods has been proofed to be at least as effective as traditional varicose vein surgery but is associated with fewer adverse events. Accordingly, the endovenous methods are exspected to replace surgery as goldstandard treatment and endovenous thermal vein ablation (Laser/Radiofrequency) is accepted by the Swiss Federal Office of Public Health for general reimbursment since 1st january 2016.

All currently available methods have their advantages and limitations, the individual selection based on the present symptoms, anatomic findings and patients' preferences is fundamental for an optimal treatment result and maximized patient satisfaction.
\end{abstract}

Key words: varicose vein treatment - endovenous ablation - laser - radiofrequency

\begin{abstract}
Résumé: Les symptômes des jambes douloureuses sont fréquents, mais leur importance est fréquemment sousestimée. Avec l'introduction de modalités de traitement endoveineuses, efficaces et minimalement invasives, le traitement des veines variqueuses s'est considérablement amélioré récemment. Chaque décision thérapeutique se base sur une anamnèse compétente, un examen clinique et une ultrasonographie avec effet Doppler. Le traitement des veines variqueuses par voie endoveineuse s'est avéré être au moins aussi efficace que la chirurgie des varices traditionnelle, mais associée à moins d'effets indésirables. Ainsi, on s'attend à ce que les méthodes endoveineuses remplacent la chirurgie comme traitement étalon et le remboursement complet de l'ablation veineuse thermique par voie endoveineuse (par laser/radiofréquence) a été accepté par l'Office Fédéral Suisse de la Santé Publique, ceci à partir du 1.1.2016.
\end{abstract}

Mots-clés: traitement des veines variqueuses - ablation endoveineuses - laser - radiofréquence

Mit Anerkennung der thermischen endovenösen Venenablationsverfahren zur Behandlung der Stammveneninsuffizienz durch das Bundesamt für Gesundheit zum 1. Januar 2016 stehen dem Kliniker nun auch offiziell Behandlungsalternativen zur klassischen chirurgischen Krampfaderoperation zur Verfügung. Die Auswahl der richtigen Krampfadertherapie ist facettenreich und erfor- dert ein eingehendes Verständnis sowohl in Hinblick auf die Symptome und Bedürfnisse des Patienten als auch be-

\section{Im Artikel verwendete Abkürzungen:}

BAG Bundesamt für Gesundheit

CEAP Clinic, etiology, anatomy, pathophysiology

MOCA Mechano-chemische Ablation

PAVK Periphere arterielle Verschlusskrankheit 
züglich der anatomisch/pathophysiologisch zugrundeliegenden Quelle des Venenleidens.

Krampfadern sind in der Allgemeinbevölkerung mit einer Prävalenz von 5-30 \% sehr häufig. Zur besseren und einheitlichen Charakterisierung der chronisch venösen Insuffizienz hat sich international eine deskriptive Einteilung nach den CEAP (clinic, etiology, anatomy, pathophysiology)-Kriterien durchgesetzt [1] und löst zunehmend die in der Schweiz noch verbreitete Klassifizierung nach Widmer ab (Tab. 1).

Eine Abklärung derselben ist nicht immer zwingend (z.B. bei asymptomatischen Besenreisern, C1), bei chronisch-fortgeschrittener Morphologie und funktioneller Dysfunktion des Venensystems (C3-C6 nach CEAP) oder Komplikationen wie Variko-/Thrombophlebitis oder Varizenblutungen empfiehlt sich jedoch eine Abklärung. Aufgrund des oft chronischen und langsam progredienten Verlaufs beziehen viele Patienten ihre Beschwerden nicht auf ein allfällig vorhandenes Venenleiden. Deshalb sind sie erfahrungsgemäss häufig umso mehr über den substanziellen Gewinn an Lebensqualität nach einer Venenbehandlung erfreut.

\section{Diagnostik}

Symptome des Krampfaderleidens beinhalten Schmerzen und Spannungsgefühl, Beinschwere, Juckreiz, Brennen, Bein- bzw. Wadenkrämpfe, geschwollene Beine, Hautveränderungen und Missempfindungen. Diese Symptome können im Verlauf des weiblichen Zyklus, einer Schwangerschaft und unter exogener hormoneller Therapie verstärkt sein. Im Gegensatz zu den Beschwerden bei peripherer arterieller Verschlusskrankheit nehmen die mit dem Krampfaderleiden assoziierten Beschwerden typischerweise unter konsequenter Kompressionstherapie und/oder Beinhochlage ab. Neben der Anamnese und der klinischen Untersuchung ist heutzutage die Duplexsonografie die Bildgebung der Wahl zur Beurteilung der Varikose der unteren Extremitäten [2-4] (Abb. 1). Neben ihrer diagnostischen Zuverlässigkeit ist sie kostengünstig, nicht

Tabelle 1. Klinische Stadieneinteilung der chronisch venösen Insuffizienzen nach CEAP-Klassifikation

Klinische Klassifikation nach CEAP

C 0 Unauffällige Inspektion/Palpation

C 1 Teleangiektasie, retikuläre Venen ( $(3 \mathrm{~mm})$

C 2 Varikose $(>3 \mathrm{~mm})$

C 3 Ödem

C 4 Hautveränderungen

a Pigmentierung, Ekzem

b Lipodermatosklerose, Atrophie blanche

C 5 abgeheiltes Ulkus

C 6 aktives Ulkus

Abk.: $\mathrm{C}=$ Clinic, $\mathrm{E}=$ Etiologie, $\mathrm{A}=$ Anatomie, $\mathrm{P}=$ Pathophysiologie

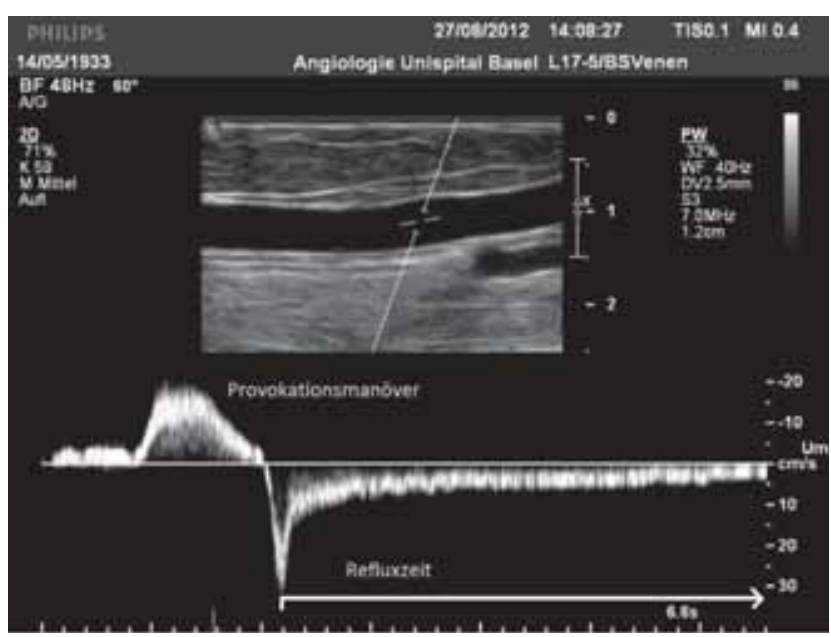

Abbildung 1. Duplexsonografischer Nachweis von Reflux. Nach Provokationsmanöver (distale Kompression) eindeutiger Nachweis eines pathologischen, lang andauernden venösen Blutrückstromes in der Vena saphena magna.

invasiv sowie nebenwirkungsfrei, und sie erlaubt nicht zuletzt die Beurteilung der perivaskulären Umgebung. Die Duplexsonografie erlaubt eine systematische und in der Regel abschliessende Beurteilung der relevanten anatomischen und funktionellen Aspekte der Venenleiden und ist wegweisend bei der Entscheidung bezüglich des Therapieplans. Vor Eingriffen am Venensystem sollte eine duplexsonografische Beurteilung durch einen fachgerecht ausgebildeten Untersucher als obligatorisch angesehen werden. In den letzten Jahren wurden im internationalen Rahmen verstärkte Bemühungen zur standardisierten Untersuchung und Interpretation unternommen. Hier sind insbesondere die Konsensus-Empfehlungen der Union Internationale de Phlébologie (UIP) bezüglich der Nomenklatur und der duplexsonografischen Methodik zu erwähnen [5-7]. Ziel der Untersuchung ist es, ein Verständnis hinsichtlich detaillierter Morphologie und spezifischer Hämodynamik der Varikose im betroffenen Bein unter Berücksichtigung der allfälligen Therapieoptionen zu gewinnen.

Eine erfolgreiche Krampfadertherapie beinhaltet in der Regel eine Elimination des oberflächlichen venösen Refluxes unter Berücksichtigung der Perforansvenen und des tiefen Venensystems.

\section{Therapieoptionen}

\section{Konservative Therapie (Kompressionsstrümpfe)}

Die älteste Therapieform bei Krampfadern ist die Kompressionstherapie, die durch äusseren Druck den Durchmesser der venösen Beingefässe verengt und dadurch auf physikalische Weise die Fliessgeschwindigkeit und den Rückstrom des Blutes in den (oberflächlichen) Venen erhöht. Entscheidend hierfür sind neben korrekt ausgemessenen und angepassten medizinischen Kompressionsstrümpfen (in der Regel der Kompressionsklasse 2 mit 
einem definierten Kompressionsdruck von 23-32 mmHg auf Knöchelniveau) auch eine ausreichende Bewegung des Patienten [8]. Kompressionsstrümpfe können nur begrenzt aktiv entstauen und unterstützen vorderhand im Sinne eines Widerlagers den durch die Muskelpumpe forcierten venösen Rückstrom. Ziele der Kompressionstherapie sind dementsprechend, Stauungssymptome zu lindern und Varizen-assoziierte Komplikationen wie z.B. eine Varikothrombophlebitis zu verhindern. Das Fortschreiten der Varikosis wird aber hierdurch nicht beeinflusst.

Entscheidender Nachteil dieser Therapie ist, dass die Kompression in der Regel dauerhaft, d.h. lebenslang durchgeführt werden muss und dass viele Patienten die Strümpfe insbesondere im Sommer als unbequem empfinden und dadurch häufig nur inkonsequent tragen. Das Anziehen kann zudem besonders für ältere Menschen schwierig sein, diverse Anziehhilfen können hier jedoch eine gewisse Erleichterung bringen und sollten aktiv angeboten werden. Bei Patienten mit peripherer arterieller Verschlusskrankheit (PAVK) und Polyneuropathie kann eine Kompressionstherapie in den meisten Fällen suffizient durchgeführt werden, eine vorherige angiologische Abklärung mit Instruktion sowie engmaschiger klinischer Kontrolle der Patienten ist aber angezeigt.

Eine Kompressionstherapie ist aktuell vor allem weiterhin die Therapie der ersten Wahl bei einer Insuffizienz der tiefen Beinvenen. Vor einer Dauerkompressionstherapie sollten hierbei jedoch potenziell behebbare Ursachen der tiefen Beinveneninsuffizienz (z.B. proximale venöse Obstruktion) und allfällige kausale Therapieoptionen (z.B. Revaskularisation/Stenting der Obstruktion) evaluiert und ausgeschlossen werden.

Bei Insuffizienz der oberflächlichen Beinvenen hat es mit Einführung der neuen, weniger invasiven Therapieoptionen in den gängigen Fachleitlinien einen Paradigmenwechsel mit nun Empfehlung zur primären Sanierung des Krampfaderleidens gegeben [2-4]. Diese beinhaltet gewöhnlich eine Behandlung der Stammvenen, namentlich der Vena saphena magna, der Vena saphena parva und der Vena saphena accessoria anterior/posterior, in speziellen Fällen aber auch von Perforansvenen.

\section{Chirurgische Therapie (Crossektomie/Stripping)}

Die traditionelle Therapie der Krampfaderbehandlung besteht in der chirurgischen Unterbindung der Stammvenen im Bereich der Einmündung in das tiefe Venensystem mittels Leistenschnitt (Crossektomie) und «Herausziehen» (Stripping) der Vene meist in Vollnarkose oder Spinalanästhesie (Abb. 2). Viele Studien konnten die Wirksamkeit der chirurgischen Therapie in der Behandlung von Krampfaderleiden zweifelsfrei belegen. Mit Einführung der modernen endovenösen Verfahren kam es in den letzten Jahren in vielen Ländern zu einem Rückgang an klassischen Krampfaderoperationen. Aufgrund der im Vergleich zur endovenösen Therapie grösseren Invasivität und damit verbundenen höheren Morbidität (Hämatome, Nervenläsionen, Infektionen) wird die chirurgische Krampf- adertherapie in neueren Richtlinien von europäischen und internationalen Fachgesellschaften mittlerweile nicht mehr uneingeschränkt als Methode der ersten Wahl betrachtet [2-4].

Von Einzelfällen abgesehen, ist bei Patienten, die Kandidaten für eine klassische Operation (Crossektomie/ Stripping) der Stammvenen sind, auch eine endovenöse Therapie möglich. Endovenöse Techniken werden in der Regel, wie allerdings heutzutage auch die Varizenoperation, problemlos ambulant durchgeführt und beinhalten entweder eine thermische, mechanische und/oder chemische Venendestruktion, bei Bedarf kombiniert mit einer Miniphlebektomie und/oder Sklerotherapie von Seitenästen bzw. retikulären Varizen. Patienten mit direkt unter der Haut gelegenen kaliberstarken Stammvenen (Gefahr einer Hyperpigmentierung im behandelten Bereich bei endovenösen Verfahren) und/oder sehr ausgedehnten beidseitigen Seitenästen (aufgrund der Maximaldosis an Lokalanästhesie) profitieren jedoch aus unserer Sicht weiterhin von einer chirurgischen Sanierung. Ebenso qualifizieren gewisse Patienten nicht für einen Eingriff in Lokalanästhesie. Hier bietet sich ebenfalls weiterhin die klassische Operation an.

\section{Endovenöse Therapieverfahren}

\section{Thermische endovenöse Venenablation (Laser/ Radiofrequenz)}

Das Prinzip der endovenösen Laser- und RadiofrequenzVenenablation beruht auf einer lokalen thermischen Zerstörung der Venenwand mit konsekutiver Schrumpfung und Fibrosierung der behandelten Vene (Abb. 3). Im Ge-

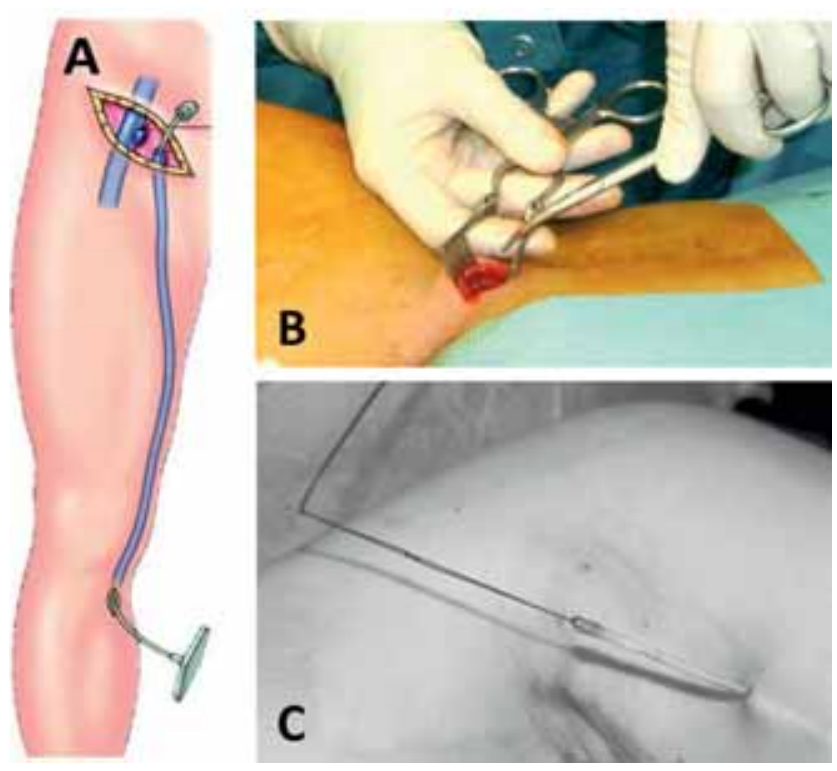

Abbildung 2. Crossektomie/Stripping. A) Schemazeichnung. Ligatur der Vena saphena im Mündungsbereich mittels Leistenschnitt, anschliessend Stripping der Vene nach distal. B) Operativer Situs bei Crossektomie. C) Invaginations-Stripping der Vena saphena magna. (Quellen: A):Vascutek, Inchinnan, Scotland UK; B/C): eigene) 

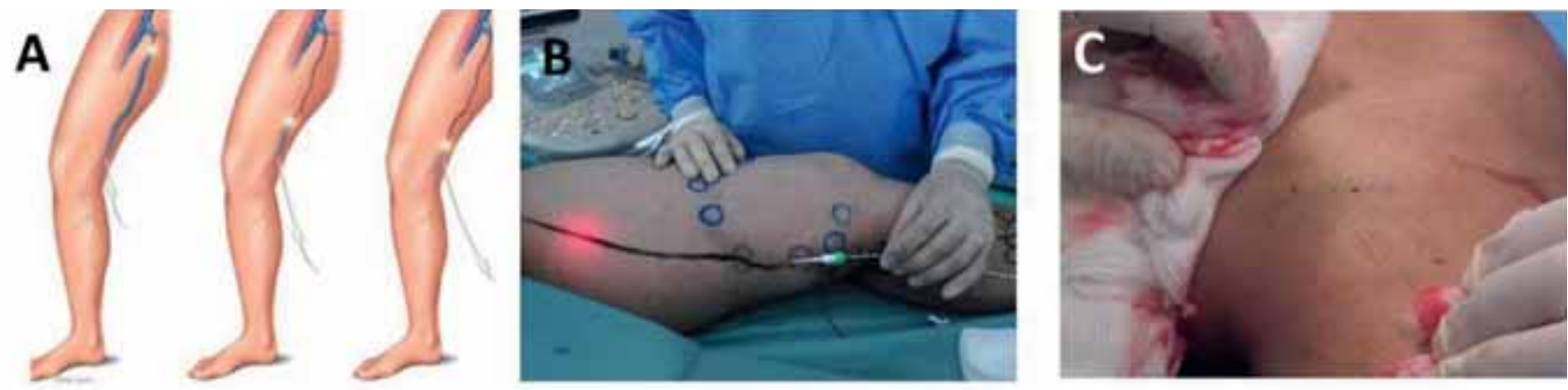

Abbildung 3. Thermische Ablation. A) Nach ultraschallgesteuerter Einlage des Katheters bis in den Crosse-Bereich langsamer Rückzug des Katheters unter Hitzeapplikation (Laser/Radiofrequenz) mit thermischer Verödung der Vene. B) Endovenöse Laserintervention: Gut zu sehen ist der rote Pilotstrahl der Lasersonde. C) Punktionsstelle nach erfolgter Laser-Ablation der Vena saphena magna. (Quellen: A): www.rwrad.com; B): eigene)

gensatz zur traditionellen Krampfaderchirurgie (Crossektomie/Venenstripping) ist bei diesen modernen Verfahren ein Schnitt in der Leiste oder Kniekehle nicht mehr notwendig. Nach ultraschallgesteuerter Punktion der Vene wird über einen 1,7 (15G-grauer Venflow) bis $3 \mathrm{~mm}$ (7-French-Schleuse) grossen Zugang ein Mikrokatheter in die Vene eingelegt. Nach Applikation einer lokalen Kühlund Anästhesielösung (Tumeszenz) entlang der Vene (Abb. 4) wird anschliessend der Katheter kontrolliert zurückgezogen und die Vene im Verlauf thermisch verödet. Endovenöse thermische Verfahren würden wir nicht in einer anderen Narkoseform als der lokalen Tumeszenzanästhesie durchführen, da die Tumeszenzlösung neben der Anästhesie auch einen effektiveren Kontakt der Sonde mit der Venenwand und einen Schutz des umliegenden Gewebes vor übermässiger Hitzeentwicklung bewirkt. Von zentraler Bedeutung ist hierbei die kontinuierliche Kontrolle der therapeutischen Schritte mittels Ultraschall, wodurch die fundierte Beherrschung desselben ganz entscheidend für den vollständigen Erfolg und die Sicherheit der Intervention ist. Je nach Schmerzperzeption wird die Applikation der Tumeszenzanästhesie als unterschiedlich schmerzhaft empfunden, die kurzzeitige Inhalation eines Sauerstoff-Lachgasgemisches wurde diesbezüglich als effektive Möglichkeit zur Analgesie beschrieben [9].

Mehrere randomisierte Studien haben eine mindestens gleich gute Wirksamkeit der thermischen Katheterablation im Vergleich zur konventionellen Chirurgie bei gleichzeitig geringeren Nebenwirkungen und schnellerer Rückkehr zu normalen Alltagsaktivitäten belegt [10-12]. Mit allen Methoden ist grundsätzlich von einer persistierend vollständigen Verschlussrate der behandelten Vene im Bereich von $>90-95 \%$ auszugehen [13,14]. Mit Entwicklung neuerer Katheter und zunehmender Erfahrung ist mittlerweile auch die gezielte Therapie von relevanten Perforansvenen wie auch ein effektiver Verschluss von sehr grossen Venen bis $30 \mathrm{~mm}$ Durchmesser mit diesen Verfahren technisch möglich. Bezüglich der Langzeitresultate ( $>5$ Jahre) liegen aktuell noch relativ wenig randomisierte Studiendaten betreffend endovenösen Katheterverfahren vor. Teilweise beobachtete sonografisch höhere Rezidivraten liegen möglicherweise in der Verwendung von Ablationssystemen der ersten Generation begründet, wobei bezüg- lich der klinischen Symptome keine signifikanten Unterschiede zur Chirurgie bestanden $[15,16]$.

Dementsprechend wurden die thermischen endovenösen Ablationsverfahren durch das BAG nun ebenfalls zur Behandlung der Stammvenen anerkannt und als vergütungspflichtige Leistungen der obligatorischen Krankenpflegeversicherung per 1. Januar 2016 in den Leistungskatalog aufgenommen.

Mit der Kostenübernahme durch die Krankenkassen ist ein zunehmender Wandel hin zu den endovenösen Verfahren auch in der Schweiz zu erwarten (Anteil in den USA $>90 \%$ ).

\section{Chemische Ablation (Sklerotherapie)}

Die chemische Ablation kann in vielen Formen durchgeführt werden. Im Allgemeinen wird hierunter eine ultraschallgesteuerte Schaumsklerosierung mittels eines Sklerosans - in der Schweiz vor allem Aethoxysklerol in unterschiedlichen Konzentrationen - verstanden. Zur Behandlung der Stammvenen wird hierbei ein Aethoxysklerol/Luft-Gemisch entweder via Spritze oder Katheter in die Vene ultraschallkontrolliert injiziert. Das Sklerosans bewirkt neben einem Vasospasmus eine Venenendothelschädigung, wodurch es zur Thrombosierung und letztendlich zur Fibrosierung des behandelten Venenabschnit-
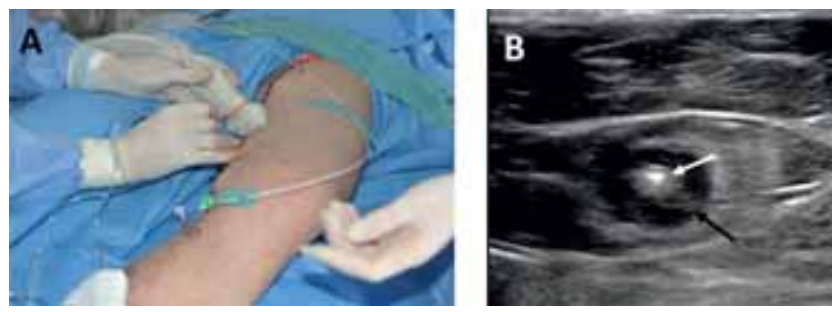

Abbildung 4. Tumeszenzanalgesie. Bei den thermischen endovenösen Verfahren (Laser/Radiofrequenz) wird ultraschallgesteuert eine Kühl-/Anästhesielösung (=Tumeszenzlösung) entlang der Vene appliziert. Die Tumeszenzlösung (schwarzer Pfeil) bewirkt eine Kompression der Vene und damit einen optimalen Kontakt der Venenwand mit der Laser-/Radiofrequenzsonde (weisser Pfeil) und schützt das umliegende Gewebe während der Hitzeapplikation. (Quelle: eigene) 

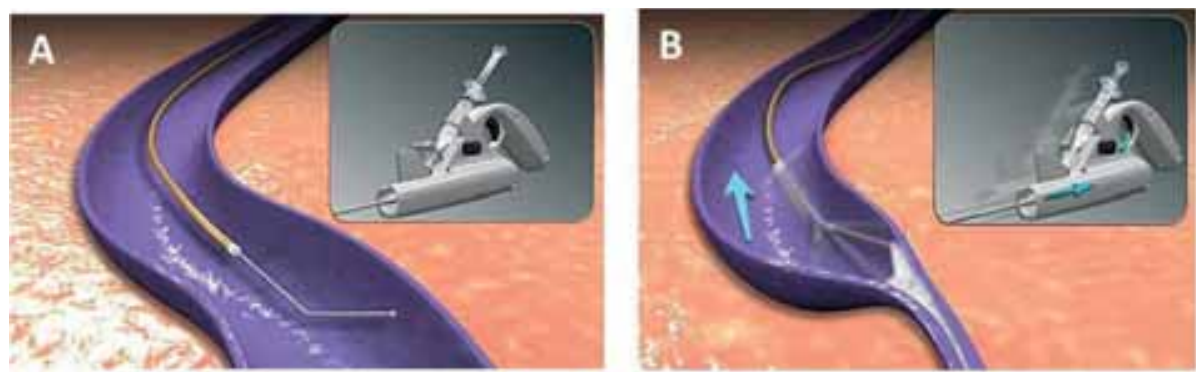

Abbildung 5. Mechano-chemische Ablation (MOCA). A/B) Nach ultraschallgesteuerter Platzierung mische Verödung hervorruft . C) Ultraschallbild während der Behandlung mit Darstellung der rotierenden Dra bereits verschlossener behandelter Vene. (Quellen: A/B): Sapheon Inc.; B/C): eigene)

tes kommt. Eine relativ häufige Nebenwirkung der Sklerotherapie sind eine Thrombophlebitis und konsekutive Hyperpigmentation entlang der behandelten Vene durch Hämosiderinablagerung. Eine lokale Thrombusexprimierung ein bis drei Wochen nach Behandlung kann die Entzündungsreaktion und Hyperpigmentation, die in der Regel temporär ist, deutlich reduzieren. Vorteile der Sklerotherapie im Vergleich zu den anderen Verfahren sind neben den geringeren Kosten die relativ einfache und schnelle Anwendung. Aufgrund der im Vergleich geringeren Effektivität ( $80 \%$ Verschlussrate) wird die Sklerotherapie nicht als Methode der ersten Wahl für die Behandlung von Stammvenen, sondern vorderhand zur Behandlung von Seitenästen und/oder einer Rezidivvarikose empfohlen [2-4]. Hier eignet sich die ultraschallgesteuerte Schaumsklerotherapie im Speziellen zur Behandlung von inguinalen oder poplitealen Crossenrezidiven (Neocrosse) nach operativer Crossektomie, insbesondere multifokale, torquierte und mässigkalibrige Neocrossen lassen sich sicher, zuverlässig und schonend verschliessen, womit in den meisten Fällen heute auf eine operative Crossenrevision verzichtet werden kann.

\section{Mechano-chemische Ablation (MOCA-Clarivein)}

Das MOCA-Kathetersystem ist eine Weiterentwicklung der Sklerotherapiemethode und basiert auf einem zweifachen Wirkprinzip. Nach ultraschallgesteuerter Platzierung bewirkt eine rotierende Katheterspitze eine mechanische Schädigung des Endothels, während das gleichzeitig während des Rückzugs injizierte Sklerosans zusätzlich eine chemische Verödung hervorruft (Abb. 5). Die Effektivität konnte dadurch gegenüber der Sklerotherapie gesteigert werden und erreicht eine im Vergleich zu den thermischen Verfahren zwar leicht geringere, aber ebenfalls hohe Verschlussrate (um 90\%) in der Behandlung von Stammvenen $[17,18]$. Da bei dieser Methode keine Hitze appliziert wird, ist keine lokale Kühlflüssigkeit/Betäubung entlang der Vene mehr notwendig und das Risiko von Haut- und Nervenschäden praktisch ausgeschlossen. Dies ermöglicht auch die Therapie von sehr distalen Abschnitten der Vena saphena magna/parva am Unterschenkel ohne erhöh- tes Risiko einer Nervenverletzung. Bei Patienten mit Asthma, symptomatischem offenem Foramen ovale und Migräne wird diese Methode aufgrund der relativ grossen Menge an Sklerosansmittel nicht empfohlen. Aufgrund

\section{Key messages}

- Venöse Beinbeschwerden sind häufig, werden aber oft unterschätzt.

- Grundlage jeder Behandlung ist eine kompetente Anamnese, die klinische Untersuchung und eine Duplexsonografie.

- Ambulante endovenöse Verfahren werden bei mindestens gleich hoher Effektivität, aber geringeren Nebenwirkungen die traditionelle Chirurgie als Methode der ersten Wahl in der Behandlung von Stammvenen zunehmend ablösen.

- Seit 1. Januar 2016 sind die endovenösen thermischen Verfahren (Laser/Radiofrequenz) zur Stammvenentherapie durch das BAG anerkannt und vergütungspflichtige Leistungen in der Grundversorgung.

- Alle Methoden haben Vor-und Nachteile bzw. Limitationen; eine individuelle Auswahl anhand der vorhandenen Symptome, anatomischen Befunde und Patientenpräferenzen ist für ein optimales Ergebnis und maximale Patientenzufriedenheit elementar.

\section{Lernfragen}

1. Welche Methoden sind zur Behandlung einer Stammveneninsuffizienz vergleichbar effektiv? (Einfachauswahl, 1 richtige Antwort)
a) Crossektomie/Stripping und Schaumsklerosierung
b) Radiofrequenz und Schaumsklerosierung
c) Laser und Schaumsklerosierung
d) Crossektomie/Stripping, Radiofrequenz und Laser

2. Welche Methoden benötigen weder eine Voll- oder Teilnarkose bzw. Tumeszenzanalgesie? (Einfachauswahl, 1 richtige Antwort)
a) Crossektomie/Stripping
b) Radiofrequenz/Laser
c) Venaseal/Clarivein
d) Miniphlebektomie 

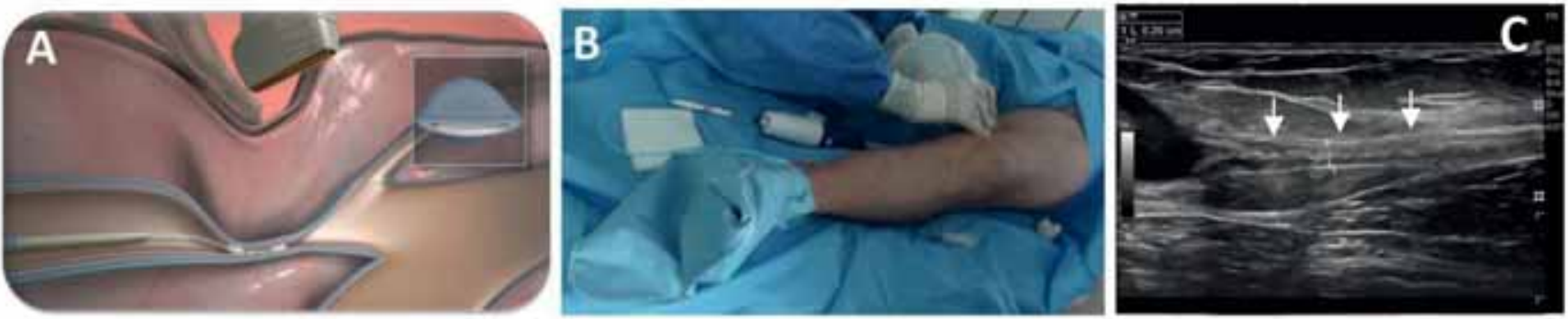

Abbildung 6. VenaSeal. A/B) Mittels Ultraschall-Kontrolle gezielte Injektion des Cyanoacrylat-Gewebeklebers und Verschluss der Vene unter manueller Kompression. C) Sonografisch vollständig verschlossene Vena saphena magna unmittelbar nach der VenaSeal-Intervention. (Quelle: A): Sapheon Inc.; B/C): eigene)

der noch unzureichenden Datenlage ist dieses Verfahren zurzeit noch nicht als obligatorische Leistung durch die Krankenkassen vergütungspflichtig.

\section{Cyanoacrylat-Gewebekleber (VenaSeal)}

Die neueste und besonders minimal-invasive Methode zur Behandlung von Stammvenen ist die Obliteration mittels Cyanoacrylat, einem speziellen «Sekundenkleber». Hierbei wird ultraschallkontrolliert ein spezieller Teflonkatheter in die Vene eingelegt und mittels Applikator die Vene von innen über den Katheter mit insgesamt ca. $1 \mathrm{ml}$ des Gewebeklebers verklebt (Abb. 6). Klare Vorteile der Methode sind, dass sowohl eine örtliche Betäubung (Tumeszenz) entfällt und die Vene in allen Abschnitten d.h. bis ganz distal ohne Risiko einer Nervenverletzung behandelt werden kann. Auch das Tragen von Kompressionsstrümpfen entfällt und die Patienten können in der Regel unmittelbar nach dem Eingriff die normalen Tätigkeiten wieder aufnehmen. Eine erste europäische Studie (eSCOPE) konnte eine Verschlussrate von 92,9\% nach zwölf Monaten demonstrieren, neueste Daten einer randomisierten Studie aus den USA (VeClose) an 222 Patienten zeigten sogar eine noch höhere Verschlussrate von $98,9 \%$ nach sechs Monaten [19,20]. Auch die erfolgreiche minimalinvasive Behandlung von Perforansvenen wurde mit dieser Methode beschrieben [21]. Aufgrund der sehr geringen Nebenwirkungen (vorderhand temporäre lokale Entzündungsreaktionen) und der hohen Erfolgsrate ist diese Therapieform trotz aktuell noch fehlenden Langzeitdaten und höheren Kosten somit eine zunehmend interessante Therapieoption für Patienten mit Krampfaderleiden, wegen der eingeschränkten Datenlage aber ebenfalls noch keine Pflichtleistung der Krankenkassen.

\section{Schlussfolgerung}

Die Behandlung von Krampfadern hat mit der Entwicklung von effektiven, minimal-invasiven endovenösen Methoden in den letzten Jahren grosse Fortschritte gemacht. Entscheidend ist aber weiterhin eine sorgfältige und genaue Diagnosestellung inklusive Duplexsonografie, sodass je nach Fortschritt der Erkrankung für jeden Patienten die am besten geeignete Methode ausgewählt werden kann.

\section{Bibliografie}

1. Rabe E, Pannier F: Clinical, aetiological, anatomical and pathological classification (CEAP): gold standard and limits. Phlebology 2012; 27 (Suppl 1): 114-118.

2. Gloviczki P, Comerota AJ, Dalsing MC, et al.: The care of patients with varicose veins and associated chronic venous diseases: clinical practice guidelines of the Society for Vascular Surgery and the American Venous Forum. J Vasc Surg 2011; 53: 2S-48S.

3. Marsden G, Perry M, Kelley K, Davies AH: Diagnosis and management of varicose veins in the legs: summary of NICE guidance. BMJ 2013; 347: f4279.

4. Wittens C, Davies AH, Bækgaard N, et al.: Editor's choice - management of chronic venous disease: clinical practice guidelines of the European Society for Vascular Surgery (ESVS). Eur J Vasc Endovasc Surg 2015; 49: 678-737.

5. Cavezzi A, Labropoulos N, Partsch H, et al.: Duplex ultrasound investigation of the veins in chronic venous disease of the lower limbs -UIP consensus document. Part II. Anatomy. Vasa 2007; 36: 62-71.

6. Coleridge-Smith P, Labropoulos N, Partsch H, Myers K, Nicolaides A, Cavezzi A: Duplex ultrasound investigation of the veins in chronic venous disease of the lower limbs - UIP consensus document. Part I. Basic principles. Vasa 2007; 36: 53-61.

7. De Maeseneer M, Pichot O, Cavezzi A, et al.: Duplex ultrasound investigation of the veins of the lower limbs after treatment for varicose veins - UIP consensus document. Eur J Vasc Endovasc Surg 2011; 42: 89-102.

8. Eberhardt RT, Raffetto JD: Chronic venous insufficiency. Circulation 2014; 130: 333-346.

9. Meier TO, Jacomella V, Clemens RKJ, Amann-Vesti B: Nitrous oxide/oxygen inhalation provides effective analgesia during the administration of tumescent local anaesthesia for endovenous laser ablation. Vasa 2015; 44: 473-478.

10. Nesbitt C, Bedenis R, Bhattacharya V, Stansby G: Endovenous ablation (radiofrequency and laser) and foam sclerotherapy versus open surgery for great saphenous vein varices. Cochrane Database Syst Rev 2014; 7: CD005624.

11. Brittenden J, Cotton SC, Elders A, et al.: A randomized trial comparing treatments for varicose veins. N Engl J Med 2014; 371:1218-1227.

12. Murad MH, Coto-Yglesias F, Zumaeta-Garcia M, et al.: A systematic review and meta-analysis of the treatments of varicose veins. J Vasc Surg 2011; 53: 49S-65S.

13. Bisang U, Meier TO, Enzler M, Thalhammer C, Husmann M, Amann-Vesti BR: Results of endovenous ClosureFast treatment for varicose veins in an outpatient setting. Phlebology 2012; 27: 118-123.

14. von Hodenberg E, Zerweck C, Knittel M, Zeller T, Schwarz T: Endovenous laser ablation of varicose veins with the $1470 \mathrm{~nm}$ diode laser using a radial fiber - 1-year follow-up. Phlebology 2013; 30: 86-90.

15. Rasmussen L, Lawaetz M, Bjoern L, Blemings A, Eklof B: Randomized clinical trial comparing endovenous laser ablation 
and stripping of the great saphenous vein with clinical and duplex outcome after 5 years. J Vasc Surg 2013; 58: 421-426.

16. Gauw SA, Lawson JA, van Vlijmen-van Keulen CJ, Pronk P, Gaastra MTW, Mooij MC: Five-year follow-up of a randomized, controlled trial comparing saphenofemoral ligation and stripping of the great saphenous vein with endovenous laser ablation $(980 \mathrm{~nm})$ using local tumescent anesthesia. J Vasc Surg 2015; 63: 420-428.

17. Vun SV, Rashid ST, Blest NC, Spark JI: Lower pain and faster treatment with mechanico-chemical endovenous ablation using Clarivein ${ }^{\oplus}$. Phlebology 2015; 30: 688-692.

18. Deijen CL, Schreve MA, Bosma J, et al.: Clarivein mechanochemical ablation of the great and small saphenous vein: Early treatment outcomes of two hospitals. Phlebology 2016; 31:192-197.

19. Morrison N, Gibson K, McEnroe S, et al.: Randomized trial comparing cyanoacrylate embolization and radiofrequency ablation for incompetent great saphenous veins (VeClose). $J$ Vasc Surg 2015; 61:985-994.

20. Almeida JI, Javier JJ, Mackay EG, Bautista C, Cher DJ, Proebstle TM: Two-year follow-up of first human use of cyanoacrylate adhesive for treatment of saphenous vein incompetence. Phlebology 2015;30: 397-404.

21. Toonder IM, Lam YL, Lawson J, Wittens CH: Cyanoacrylate adhesive perforator embolization (CAPE) of incompetent perforating veins of the leg, a feasibility study. Phlebology 2014; 29: 49-54.
Manuskript eingereicht: 8.2.2016

Manuskript nach Revision angenommen: 26.2.2016

Interessenskonflikt: Die Autoren erklären, dass kein Interessenskonflikt besteht.

\section{PD Dr. med. Heiko Uthoff}

Facharzt für Angiologie und Allgemeine Innere Medizin Gefässpraxis am See

St. Anna im Bahnhof

Zentralstrasse 1

6003 Luzern

heiko.uthoff@hirslanden.ch 